\title{
Dos discursos sobre la integración entre la ciencia de la información, la archivística y la bibliotecología
}

Two discourses on the integration between information science, archival science and library science

Radames Linares Columbié

Universidad de la Habana. Facultad de Comunicación.

Departamento de Ciencias de la Información, Cuba

radames@fcom.uh.cu

(iD https://orcid.org/0000-0003-3183-9194

Manuel Alejandro Romero

Universidad de la Habana. Facultad de Comunicación.

Departamento de Ciencias de la Información, Cuba

manuel.romero@fcom.uh.cu

(D) https://orcid.org/0000-0002-0721-623X

\section{Resumen:}

Objetivo. Se examinan los procesos de integración desarrollados entre los cuerpos de conocimientos identificados como archivística, bibliotecología y ciencia de la información, desde los discursos teóricos de los estudiosos Miguel Ángel Rendón Rojas y Armando Malheiro da Silva. Metodología. Mediante la investigación documental como herramienta, se examinan las circunstancias institucionales, profesionales, epistemológicas y científicas que condicionaron los escenarios que sirvieron de plataforma para la aparición de las perspectivas disciplinares e interdisciplinares que marcaron la historia del campo informacional y las propuestas de los autores que se examinan. Resultados. Se discuten y detallan las argumentaciones de Rendón Rojas y Silva que retomaron la discusión disciplinar en el campo informacional proponiendo sus lecturas particulares. Conclusiones. Se concluyó que el complejo proceso de integración es explicable por una coyuntura económica, científica, informacional y tecnológica existente desde los finales del siglo pasado, que sirvió de contexto impulsor del fenómeno que desarrollaron desde sus miradas Rendón Rojas y Silva.

PaLABRAS ClaVE: Integración, Archivística, Bibliotecología, Ciencia de la Información.

\section{AbStract:}

Objective. The integration processes developed between the bodies of knowledge identified as archival science, library science and information science are examined, from the theoretical discourses of the scholars Miguel Ángel Rendón Rojas and Armando Malheiro da Silva. Methodology. Through documentary research as a tool, the institutional, professional, epistemological and scientific circumstances that conditioned the scenarios that served as a platform for the emergence of disciplinary and interdisciplinary perspectives that marked the history of the informational field and the perspectives of the authors are examined. Results. The arguments of Rendón Rojas and Silva, who took up the disciplinary discussion in the informational field, are discussed and detailed, proposing their particular readings. Conclusions. It was concluded that the complex integration process is explained by an economic, scientific, informational and technological situation that has existed since the end of the last century, which served as a driving context for the phenomenon that Rendón Rojas and Silva developed from their point of view.

KEYWORDS: Integration, Archival science, Library science, Information science.

\section{INTRODUCCIÓN}

Los cuerpos de conocimiento identificados con las denominaciones de archivística, bibliotecología y ciencia de la información aparecieron como tales en el escenario académico-profesional entre los siglos XIX 
y XX. La materialización de estos procesos transitó desde las circunstancias que los enmarcaron como entidades con una relativa autonomía, hasta momentos en los cuales se desarrollan posturas que sustentan visiones diferentes a la establecida en los periodos fundacionales de estos espacios.

La instauración de estos saberes fue congruente con las diversas formas establecidas en su tiempo para organizar y presentar la producción de conocimientos de la realidad; por ello, la archivística y la bibliotecología se fundaron como disciplinas que estudiaban determinadas parcelas del escenario informacional en uno y otro caso, acorde con las reglas dominantes en ese tiempo, donde el conocimiento parcelado de la realidad de uno u otro aspecto era la tónica. De otra parte, debe anotarse que la ciencia de la información desde su instauración en la segunda mitad de la centuria pasada se autocalificó como interdisciplinaria, modalidad de organización disciplinar propia de nuevas circunstancias muy diferentes a las preponderantes en el siglo XIX.

Disciplinas o interdisciplinas son términos que a lo largo de la historia de estas materias han pretendido subrayar las cercanías y distancias que las distinguen; no obstante, la práctica histórica que ha acompañado su existencia ha evidenciado en momentos específicos, la fragilidad de sus fronteras o la marcada cercanía de sus quehaceres distintivos; es precisamente el examen de esta problemática el propósito de este texto, donde dos vocablos, entre otros, indicaran la ruta: convergencia e integración.

Es esta una temática de estudio de varios autores (Araújo, 2012; Ortega, 2004; Rendón Rojas, 2016; Silva, 2017), pero este texto en el examen que se pretende serán objeto de análisis:

- la conformación de la archivística y la bibliotecología como disciplinas en el marco del siglo de la gran explosión disciplinar.

- el momento del gran giro epocal de la segunda mitad del siglo XX, donde se gesta e impulsa un nuevo espacio de conocimiento con pretensiones no disciplinares: la Ciencia de la Información.

- las posturas, de dos de los estudiosos más relevantes del campo informacional en estos tiempos: Rendón Rojas y Silva, ante el fenómeno de la convergencia e integración de las materias informativas

En consecuencia, el estudio se propone aproximarse a las interconexiones distintivas de los saberes informacionales y debe ayudar a comprender mejor las particularidades de una de las facetas del campo informacional.

\section{Metodología}

El acercamiento que se realiza, dadas sus características, se sustenta en la investigación documental como herramienta metodológica. Este examen utiliza las fuentes bibliográficardecuadas que permiten delimitar las circunstancias institucionales, profesionales, epistemológicas y científicas que condicionaron los escenarios que sirvieron de plataforma para la aparición de las perspectivas disciplinares e interdisciplinares que marcaron la historia del campo informacional y las propuestas de los autores que se examinan. El anlisis puntual de los discursos teóricos de Rendón Rojas y Silva se sustenta en la producción científica de estos autores referida específicamente a la problemátic que es centro de gravedad de la revisión que se propone, a saber, el proceso de integración de los campos de conocimiento informacionales.

\section{Resultados y Discusión}

\subsection{Archivística, bibliotecología y explosión disciplinar}

Cualquier aproximación al devenir histórico de la archivística, la bibliotecología y la ciencia de la información tiene una inexorable plataforma de arranque; instituciones que distinguen a dos de estos 
espacios las anteceden en el tiempo: las bibliotecas y los archivos. Es decir... "las instituciones y servicios Archivo, Biblioteca... están en la raíz y en la designación de las respectivas disciplinas profesionales" (Silva, 2015, p. 104). Con otras palabras, el surgimiento de los archivos y las bibliotecas es el ineludible punto de partida de esta mínima aproximación al estudio de las disciplinas mencionadas.

Los primeros tiempos de la existencia histórica de los "archivos" y "bibliotecas", a saber: antigüedad y edad media, fueron momentos donde no hubo distinciones significativas entre esas instituciones, usualmente compartían los mismos espacios físicos e iguales profesionales (Ortega, 2009). La diferencia entre esas instituciones comenzó a evidenciarse con la llegada de la modernidad (enmarcada entre el siglo XV y la segunda mitad del XX), instante de notables episodios, entre los que sobresalen: la creación de los Estados nacionales, la difusión de la imprenta, las crisis religiosas, el racionalismo filosófico, las revoluciones políticas, las revoluciones técnicas, la revolución científica, entre otros. Estos eventos son representativos de la confrontación con el viejo orden medieval y las muestras iniciales de la emergencia de la modernidad burguesa, que impactaron de manera decisiva en la separación que se estableció entre archivos y bibliotecas.

Esta alteración cualitativa es también consecuencia de la expansión bibliográfica generada por la creación de la imprenta en el siglo XV y su impacto en las bibliotecas de ese momento, al igual que el aumento de las actividades administrativas de los estados nacionales modernos que generaron un crecimiento de los archivos como instituciones fundamentales para las gestiones político-administrativas e hicieron más evidente la separación entre esas entidades (Tanus, 2016). De manera que...

los desarrollos de las bibliotecas y los archivos hasta el siglo XIX hicieron muy clara la necesidad de ir más allá de lo puramente instrumental de la labor bibliotecaria y archivera. La creciente significación cultural, social y económica de estas instituciones, impulsaron los intentos por construir específicos espacios de conocimiento que abordaran la problemática de las bibliotecas y archivos, que trascendiera la tradicional empiria que tipifican sus actividades; y en consecuencia se instrumentan concepciones en torno a la necesidad de crear áreas particulares de estudio (Linares Columbié, 2018, p. 9).

Si bien lo antes expuesto examina una de las dimensiones del proceso de constitución formal de la bibliotecología y la archivística, es necesario anotar otros aspectos que facilitan la comprensión de este asunto, entre otros, se debe subrayar que el siglo XIX como instante fundacional de estas materias, es también el momento de un sustantivo auge de las ciencias naturales y del surgimiento de las ciencias sociales, esta explosión del saber científico se estructuro a través de diversas formas de organización, difusión y producción de conocimientos generados en los más diversos campos, la más representativa en esa época, fueron las denominadas disciplinas.

$\mathrm{Al}$ igual que, durante el siglo XIX desde el seno de la archivística se formulan los principios de procedencia y de orden natural de los documentos, y es también el siglo en el que la bibliotecología genera una pléyade de reglas, normas y leyes. Ambas con el objetivo de dialogar con la imperante filosofía positivista, las cual impone la enunciación de verdades absolutas, contrastables y mensurables, como elementos distintivos de toda disciplina.

En consecuencia, se entiende que una disciplina es un segmento de conocimiento, del acervo de conocimientos de la humanidad, convertido en singular, distinto. Fragmentar y parcelar el examen de la realidad es el rasgo que por excelencia distingue a las disciplinas. Además, en ellas habría que incluir a una comunidad, una determinada práctica, una tradición, un conjunto particular de valores y creencias, un dominio, una modalidad de investigación y una estructura conceptual (Morin, 2002). La archivística y la bibliotecología se fundan y se desarrollan desde esta perspectiva disciplinar, donde asumen todas las características propias de esa forma organizativa del saber y tendrán como centro de gravedad, en uno y otro caso, a los archivos y a las bibliotecas.

Sobre la base de las consideraciones anteriores, se puede resumir que la constitución disciplinar de la archivística y la bibliotecología desde el siglo XIX enfatiza el alejamiento entre los "archivos" y las "bibliotecas", distanciándose estas instituciones de los tiempos de acercamiento de sus anos fundacionales y serán otras circunstancias las que favorecerán un retorno a los tiempos de posible "integración”. 


\subsection{La ciencia de la información y el gran giro de la segunda mitad del siglo XX}

En efecto, la disciplinarización del conocimiento fue capaz de expresar las urgencias y necesidades de una época determinada, es decir, el desarrollo del conocimiento y el progreso tecnológico de esos años se debió, en gran parte, a la explosión cuantitativa de disciplinas. Pese a ello, la complejidad de la realidad y de los problemas que de ella emanan impulsó gradualmente la necesidad de diversos acercamientos entre las disciplinas con el fin de lograr su estudio.

Desde la segunda mitad del siglo XX se inicia un proceso de cambios en todos los terrenos, que va a tener también su manifestación en los espacios centrados en la organización y producción de conocimientos en todos los saberes. Los rasgos típicos del esquema disciplinar, comienzan a mostrar su incapacidad para responder a problemas y circunstancias de una época nueva. Una de las nuevas opciones disciplinares es la interdisciplinariedad, respuesta a los tiempos que emergen; sus orígenes se pueden encontrar bastante lejanos en el tiempo, a los efectos de esta aproximación sus marcos temporales se insertan en la segunda mitad del siglo XX.

La interdisciplinariedad, como nivel de integración disciplinar tiene un rasgo característico, la diversidad de aproximaciones al significado de esta práctica y concepción; en líneas generales se tiende a considerarla con una peculiaridad: como resultado de la cooperación entre disciplinas y sus interacciones (esta puede ir desde una simple comunicación de ideas hasta la mutua integración de conceptos, metodología, procedimientos, epistemología, terminología, datos y la organización de investigación y docencia en un campo suficientemente amplio); es decir, reciprocidad en los intercambios y, por consiguiente, un enriquecimiento mutuo (Guzmán Gómez, 2005).

La tónica de los estudios interdisciplinarios busca el desarrollo de actividades que se realizan con la cooperación e intercambio de varias disciplinas. Cada disciplina pone a disposición de las otras sus esquemas conceptuales, sometiéndolos a la asimilación y reformulación de los mismos, de los que resulta una integración diferente por esa reciprocidad en el intercambio.

La interdisciplinariedad será siempre una reacción alternativa al abordaje disciplinar de los objetos de estudio. Ella estaría situada donde se piensa superar la fragmentación de las ciencias y de los conocimientos por ellas producidos Es así que...

por interdisciplinariedad, deberá entonces entenderse cualquier forma de combinación entre dos o más disciplinas con vista a la comprensión de un objeto a partir de la confluencia de puntos de vista diferentes y teniendo como objetivo final la elaboración de una síntesis relativa al objeto común (Pombo, 1994, p. 71).

A su vez, la ciencia de la información fundada en los años ' 60 del pasado siglo, estuvo acompañada desde sus inicios por intentos de ser especificada. Estas caracterizaciones han sido diversas a lo largo de su corta historia, precisamente una de las denominaciones recibidas fue la de ciencia interdisciplinaria, coincidiendo en el tiempo con el movimiento interdisciplinar surgido como nueva forma de organización del conocimiento.

Es decir, la trilogía de saberes que centralizan este examen parece distinguirse por la conformación inicialmente de dos disciplinas en el siglo de la gran explosión disciplinar: archivística y bibliotecología; y, en el momento del gran giro epocal del siglo XX, se gesta e impulsa un nuevo espacio de conocimiento con otras pretensiones: la ciencia de la información.

La información, en sus diversas variantes, ha existido en todos los tiempos y en múltiples circunstancias sociales y culturales, pero solo a partir del siglo XX comienza a ser considerada como un fenómeno de gradual importancia en todos los terrenos. Una de las expresiones de la relevancia de la información en ese siglo, fue el marcado interés en la información especializada o profesional, en tanto su crecimiento e importancia para la actividad científica, tecnológica y económica adquiría progresivamente un carácter estratégico. La denominada ciencia de la información aparece en el escenario histórico precisamente con el propósito de 
centrar su atención en esa dimensión de la información, y la convierte en su objeto de estudio. (Vega-Almeid, Fernández-Molina y Linares Columbié, 2009).

Cabe agregar que, si bien 1962 es el año de constitución formal de la ciencia de la información en EE.UU, su advenimiento es el resultado de la conjunción de varios factores: geopolíticos, informacionales y tecnológicos, entre otros (Araújo, 2018). En fin, la aparición y desarrollo de la ciencia de la información es el producto de la estrecha vinculación establecida entre los cambios de la situación informacional en el contexto norteamericano y determinantes circunstancias económicas, políticas, tecnológicas y científicas.

El propósito de concebir a la ciencia de la información como un campo interdisciplinar es una intención que atraviesa la trayectoria de esta área de estudios, desde las primeras obras fundacionales (Borko, 1968). Hasta los tiempos actuales este propósito ha estado presente; así se afirma que la ciencia de la información es "ciencia interdisciplinar derivada o que está relacionada con la Matemática, la Lógica, la Lingüística, la Sicología, la Tecnología de la Computación, la Investigación Operacional, las Artes Gráficas, la Comunicación y la Bibliotecología" (Borko, 1968, p. 3).

Sin embargo, el discurso de la interdisciplinariedad en la Ciencia de la Información ofrece un cuadro inocuo del fenómeno. La interdisciplinariedad se presenta, así como una condición natural, quasi inmanente a este campo, más que como un proceso dinámico y complejo de construcción (Pérez, 2004).

Dado que el movimiento interdisciplinar surge en los años en que se funda la Ciencia de la Información, no es difícil percibir que esta nueva estrategia disciplinar daba sus primeros pasos, por tanto, asumirla significaba apropiarse de algo prácticamente desconocido, generando en el naciente campo informacional una interpretación y aplicación, frágil y polémica, por ello se afirma que:

no tuvo consecuencias epistemológicas importantes para la Ciencia de la Información en estos primeros años. Tal característica fue utilizada, sobre todo, para justificar la actuación en el campo, de personas provenientes de las más variadas disciplinas científicas (...). Estudios más rigurosos sobre la idea de interdisciplinariedad (de la necesaria existencia de un proceso teórico y conceptual de "a dos manos" entre las disciplinas involucradas) comenzaron a diagnosticar la inexistencia de prácticas interdisciplinares entre la Ciencia de la Información y las demás ciencias, en la medida en que la Ciencia de la Información "tomaba en préstamo" conceptos y métodos de otros campos, sin hacerse notar por ésta (Araújo, 2014, p. 14).

Desarrollos más recientes de la ciencia de la información han hecho visible una comprensión más atinada de la interdisciplinariedad, mostrando como este campo se nutre de conceptos y teorías provenientes de la psicología, lingüística, computación o sociología; así ha sido posible el nacimiento de los estudios métricos (tomando conceptos y visiones de la estadística y la matemática), o los estudios de usuarios (tomando conceptos y visiones de la psicología, la sociología, la antropología), entre otras.

\subsection{La ruta de la integración de los saberes informacionales}

De acuerdo con los razonamientos que se han venido realizando, es oportuno reiterar que la aspiración esencial de este texto, no es otra que la de estudiar la gradual y compleja aproximación e interacción que se ha producido entre los diversos cuerpos de conocimiento, objeto de este análisis: archivística, bibliotecología y ciencia de la información. Este proceso ha sido examinado por diversos autores que analizan este fenómeno, desde sus singulares propuestas.

Como se ha visto, se tratará de estudiar interacciones diversas, rompiendo en algunos casos las variantes organizativas de una u otra materia; es decir, desde disciplinas e interdisciplinas que interaccionan, hasta proponer nuevas formas de organización del saber informacional.

Se ha aclarado que, las prácticas bibliotecarias y archivísticas no nacieron separadas, solo desde la edad moderna estas rutinas y sus instituciones comenzaron a distanciarse, debido a factores diversos de orden tecnológico, como la invención occidental de la imprenta o acontecimientos como las revoluciones políticas y culturales de esa época, entre otros. Pese a ello, desde el siglo XIX comenzaron a revelarse señales de que 
la tendencia originaria que tipificó las disciplinas mencionadas, no dejo de estar presente, es así como la registrada como una de las primeras instituciones formadora de los profesionales propios de estos campos: la École des Chartes en 1821 fundada en Francia formó archiveros y bibliotecarios con una preparación unitaria, sin distinciones entre una y otra profesión (Silva, 2015).

También es posible identificar al surgimiento de la Documentación como un elemento expresivo del pensamiento integracionista. La propuesta de Paul Otlet rompe con las miradas tradicionales y ofrece una nueva perspectiva para la organización del conocimiento. Si bien la documentación nació con pretensiones de legitimarse como una disciplina autónoma su impacto se redujo en tiempos de la posguerra a solo algunas naciones europeas y algunas regiones de Canadá que siguen su impronta. El verdadero valor de la propuesta otleliana está en la sistematización del concepto de documento y la identificación de nuevas maneras de almacenar y recuperar información en contextos más amplios y desde perspectivas disciplinares diferente a las que ya existían (López Yepes, 1995).

De la misma manera, otra muestra significativa fue el desarrollo del movimiento integrador liderado por la UNESCO en los años '60 - '70 del siglo XX, que pretendió impulsar programas integrales de formación de profesionales de un solo tipo y asumir distancias de las clásicas formación separadas de archiveros, bibliotecarios o documentalistas.

Las tendencias integradoras de nuestra época son de alguna manera continuación de las señales mencionadas, solo que en este tiempo determinadas circunstancias van a ser factores impulsores de este proceso; en efecto, desde las últimas décadas del pasado siglo comienza a visibilizarse un giro tecnológico, científico e informacional que va a servir de soporte a cambios notables en los cuerpos de conocimientos informacionales, que las propuestas de los autores que se examinaran harán visibles.

\subsection{Armando Malheiro da Silva y su discurso sobre la integración}

Silva desde los inicios del siglo XXI plasma en numerosos artículos, textos, monografías y diccionarios su particular visión de la problemática epistemológica, histórica y formativa del campo informacional y documental de nuestro tiempo. ${ }^{1}$ De su conjunto de ideas, reflexiones y concepciones serán especialmente relevantes aquellas asociadas a su propuesta de integración de: la archivística, la bibliotecología y la ciencia de la información, ahora bien, ¿Cuál es el núcleo duro de su proyecto? ¿Cómo se fundamenta? ¿Cuáles son sus elementos distintivos?

El "núcleo" de su programa está en su creencia en la existencia de un espacio integrador que denomina ciencia de la información, y que no es igual a la information science anglosajona, aunque no niega que sea esta una de sus fuentes nutricias, así afirma: "La concepción de Ciencia de la Información, que venimos tejiendo y consolidando, es diferente...de la disciplina surgida en EE.UU, en plena sociedad posindustrial y que se centra en el procesamiento automatizado de la información científica y técnica" (Silva \& Ribeiro, 2010, p. 72).

Su ciencia de la información también "resulta de la fusión de las disciplinas prácticas Archivística, Bibliotecología y Documentación” (Silva, 2013, p. 93), es decir, desde la visión de este autor, las materias mencionadas no tienen carácter científico, sino exclusivamente práctico y técnico; de otra manera, la nueva materia es la resultante de la interacción e integración de las mencionadas disciplinas.

Dadas las condiciones que anteceden, se evidencia un nuevo carácter de este saber denominado ciencia de la información, que el autor califica como transdisciplinario, que es una etapa superior y posterior a la interdisciplinariedad no limitada a las interacciones o reciprocidad entre las disciplinas, sino que ubica esas relaciones o vínculos en el interior de un sistema total, sin fronteras establecidas entre las disciplinas (Silva, 2017). Con otras palabras: "la Ciencia de la Información es transdisciplinar, o sea, constituye un estadio epistemológico evolutivo, resultante de la interacción e integración de las disciplinas practicas citadas" (Silva \& Ribeiro, 2010, p. 73). 
En esta identificación de la ciencia de la información, Silva retoma la clásica calificación de la information science, que concebía a esta como interdisciplinaria, y también presenta al nuevo espacio de conocimiento con igual característica; porque esta materia se va a relacionar con dos campos esenciales: las ciencias de la información y comunicación, en primera instancia y, el amplio campo de las ciencias sociales aplicadas (Silva \& Ribeiro, 2010). En fin, la ciencia de la información que propone tiene un doble carácter: interdisciplinaria y transdisciplinaria.

El proyecto de ciencia de la información que nos ocupa, añade elementos singulares en su mirada integradora como: los factores explicativos de la concepción que nos propone este autor, para ello, se apoya en:

una metateoría explicativa, basada en dos paradigmas esenciales: el custodial, patrimonialista, historicista y tecnicista, que surge desde el siglo XVIII hasta la era de la información en que vivimos; y el poscustodial, informacional y científico, que remite al modo de ver, de pensar y de actuar de generaciones de cientistas y profesionales de la información, a lo largo del siglo XXI (Silva \& Ribeiro, 2010, p. 74).

En otros términos, la ciencia de la información que se pretende está condicionada por el paradigma poscustodial, científico e informacional, que postula una mutación epistemológica cuando cambia el objeto de estudio y de trabajo ("documento" por "información"); al distanciarse del estadio precientífico de otras materias informativas (archivística y bibliotecología) y de los abordajes instrumentales, prácticos y normativos de los documentos-artefactos propios del paradigma custodial y orientarse hacia la creación, comprensión y explicación del fenómeno infocomunicacional como centro del nuevo campo informacional (Silva, 2009).

\subsection{Miguel Ángel Rendón Rojas y su discurso sobre la integración}

Rendón Rojas, ${ }^{2}$ desde los años '90 del pasado siglo, materializa en numerosos artículos y monografías su amplia visión de la problemática filosófica, epistemológica e histórica del campo bibliotecológico e informacional:

cuenta con más de 8 libros publicados, además de 33 artículos científicos en importantes revistas y alrededor de 8 capítulos de libros. Ha participado en más de 50 eventos científicos.... Es considerado como un referente a nivel internacional del pensamiento informacional contemporáneo (Paz \& Frías, 2019, p. 140).

De su catálogo de ideas, reflexiones y concepciones serán especialmente relevantes, para este texto, aquellas asociadas a su propuesta de integración y convergencia de: la archivística, la bibliotecología y la ciencia de la información.

La amplitud y profundidad del pensamiento de este autor, hacen imprescindible algunas precisiones que pueden facilitar la comprensión de su particular enfoque del proceso de integración en el terreno informativo y documental. Este pensador, es por encima de cualquier otra consideración, un filósofo; una de sus principales preocupaciones es realizar un estudio filosófico del campo informativo y documental; esta dimensión de su obra se distancia de las intenciones de este texto, pese a ello, es prudente anotar esta peculiaridad.

Se debe comenzar enfatizando, su creencia en la viabilidad de la convergencia e integración de los tres cuerpos de conocimientos más representativos del campo informacional: bibliotecología, archivística y ciencia de la información; y, una de las primeras características de su postura, sería o es los varios nombres o denominaciones que tendría el espacio que propone, los más frecuentes son: ciencia bibliotecológica, bibliotecología, ciencia de la información documental, entre otros (Rendón Rojas, 2005).

Esa área de estudios que se presenta, con una u otra denominación, anota como una de sus precisiones fundamentales el espacio de la realidad en la cual se inserta y es la esencia de esta materia: denominada campo o mundo fenoménico informativo documental y se detalla que: 
dentro de ese fenómeno informativo documental encontramos objetos que lo habitan ("información", "documento", "fuente", "fondo", "lenguaje documental", "unidad de información", "tecnología de la información", entre otros), así como sujetos que actúan en él ("usuario", "profesional de la información documental", "autor", "comunidad", entre otros) y también procesos que tienen lugar ("generación”, “recolección”, “procesamiento”, “organización”, “almacenamiento”, “búsqueda, "recuperación”, "diseminación”, y "uso" de la información; "valoración de documentos”, "políticas de información”, entre otros) (Rendón Rojas, 2016, p. 18).

Dado el carácter de plataforma que tiene el "mundo informativo documental" propuesto por el autor, este indica que sus orígenes están en un denominado ser informacional; es decir, los individuos para existir necesitan crear, consumir, transmitir y crear información, es esa existencia ligada al ser humano lo que le otorga el carácter de ciencia social y humana al nuevo espacio de conocimiento (Rendón Rojas, 2013).

Con otras palabras, el núcleo central de esta área de conocimiento, denominada: ciencia bibliotecológica, bibliotecología o ciencia de la información documental, que es la parte común de las materias que convergen; es el llamado sistema de información documental formado por la interrelación de: la información, el documento, el usuario, el profesional de la información documental, y la institución informativa documental:

esta interacción es motivada por la finalidad de satisfacer las necesidades de información documental del usuario, a través de su ingreso al mundo de la información mediante los documentos proporcionados gracias a la actividad del profesional de la información documental dentro de una institución informativa documental (Rendón Rojas, 2013, p. 14).

El autor precisa que el sistema de información documental es un sistema porque ninguno de sus elementos se comprende dentro del estudio del mundo de la información documental de manera aislada. Por ejemplo, el objeto de estudio no es solo la información, sino que es una información objetivada en un documento, gestionada por un profesional de la información dentro de una institución informativa documental y que sirve para satisfacer las necesidades de información de un usuario que recurre a ese sistema precisamente para eso (Rendón Rojas, 2013).

Cabe agregar que dentro de las concepciones del autor examinado se valora el estatus del conjunto de materias involucrado, y en consecuencia se puntualiza que:

descubrimos una unidad sin perder la multiplicidad, podemos hablar de una realidad transdisciplinar, compleja, pero al mismo tiempo conservando las identidades de sus componentes. Ninguna de ellas coloniza o absorbe a las otras, sino que coexisten en un campo común. Archivística, Bibliotecología, Ciencia de la Información, Documentación y Museología se reencuentran, las cuatro tratan con documentos, con información (Rendón Rojas, 2016, p. 35).

O lo que es lo mismo, el discurso integrador que se postula subraya la existencia de un espacio transdisciplinar, con todo lo que implica esa modalidad disciplinar, pero se propone una coexistencia que respeta las identidades de las áreas de estudios informacionales particulares.

\section{Conclusiones}

La trayectoria académica e institucional del campo informacional y documental a nivel global se ha caracterizado en las dimensiones disciplinar e interdisciplinar, por fundar áreas de estudios particulares en los siglos XIX y XX con denominaciones específicas, las más frecuentes e identificadas como tales en este medio son: la archivística, la bibliotecología y la ciencia de la información.

Pese a estas especificidades, desde finales del siglo XX comienza a gestarse una fuerte tendencia que se aleja de los viejos esquemas disciplinares de otros tiempos y, se perciben posturas, tendencias y criterios que promueven e intentan fundamentar la existencia de una manifiesta convergencia e integración de las materias particulares de estudio de este campo. Este complejo proceso es explicable por una coyuntura económica, científica, informacional y tecnológica existente desde los finales del siglo pasado, que va a servir de contexto impulsor del fenómeno que se anota. 
Rendón Rojas y Silva, los autores que examina este texto, son dos de los pensadores contemporáneos más notables que analizan este fenómeno de la integración disciplinar en el campo informacional y proponen sus modelos disciplinares. La ciencia de la información de Silva y la ciencia bibliotecológica de Rendón Rojas son proyectos que revelan la tendencia que es centro de gravedad de este texto y que estos pensadores desarrollan y argumentan.

Sus visiones son lógicamente diferentes, cada uno propone y argumenta sus visiones particulares del proceso de integración en el campo informacional, sin que ello signifique que no haya lugares de encuentro, entre otros, es posible anotar sus propuestas sobre el carácter del nuevo espacio que formulan, tipificado como transdisciplinar y es esta la mejor expresión del proceso de la integración en el campo informacional desde el punto de vista de estos autores

\section{REFERENCIAS}

Araújo, C. A. Á. (2012). Proximidades conceituais entre arquivologia, biblioteconomia, museologia e ciencia da informacao. Biblionline, 8(2), 27-36. Recuperado de http://periodicos.ufpb.br/index.php/biblio/article/viewF ile/14291/8635

Araújo, C. A. Á. (2014). Fundamentos da ciência da Informação: correntes teóricas e o conceito de informação. Perspectivas em gestão \& conhecimento, 4(1), 57-79. Recuperado de http://periodicos.ufpb.br/ojs/index.php/p gc/article/viewFile/19120/10827

Araújo, C. A. Á. (2018). Um Mapa da Ciência da Informação: história, subáreas e paradigmas. ConCI: convergências em ciência da informação, 1(1), 45-70. Recuperado de https://seer.ufs.br/index.php/conci/article/view/9341

Borko, H. (1968). Information science: what is it? American documentation, 19(1), 3-5.

Guzmán Gómez, M. (2005). El fenómeno de la interdisciplinariedad en la ciencia de la información: contexto de aparición y posturas centrales. Acimed, 13(3). Recuperado de http://www.bvs.sld.cu/revistas/aci/vol13_3_05/ aci05305.pdf

Linares Columbié, R. (2018). Los profesionales de la información en Cuba y su formación. Bibliotecas. Anales de investigación, 14(1), 63-72. Recuperado de https://dialnet.unirioja.es/servlet/articulo?codigo=6312037

López Yepes, J. (1995). La documentación como disciplina: teoría e historia. Madrid: EUNSA.

Morin, E. (2002). La cabeza bien puesta. Repensar la reforma, reformar el pensamiento. Basespara una reforma educativa. Buenos Aires: Nueva Visión.

Ortega, C. D. (2004). Relações históricas entre biblioteconomia, documentação e ciência da informação. DataGramaZero, 5(5), 1-29. Recuperado de http://www.dgz.org.br/out04/F_I_art.htm

Ortega, C. D. (2009). A documentação como uma das origens da Ciência da Informação e base fértil para sua fundamentação. Brazilian journal of information science, 3(1), 3-35, Recuperado de http://portaldeperiodicos. eci.ufmg.br/index.php/pci

Paz, L. E. y Frías, M (2019). Discursos de Miguel Ángel Rendón Rojas en la revista Investigación bibliotecológica. SAPIENTIAE: ciências sociais, humanas e rngenharias. 5(1), 138-156 Recuperado de https://dialnet.unirioja.e s/descarga/articulo/7052371.pdf

Pérez, M. (2004). Ciencia de la Información: fisuras en el laberinto de la interdisciplina. En: Memorias del Congreso Internacional de Información INFO 2004. La Habana: Instituto de Información Científico y Tecnológico.

Pombo, O. (1994). Contribuição para um vocabulário sobre interdisciplinaridade. In O. Pombo, H. Guimaraes e T. Levy. Interdisciplinaridade: reflexão e experiência (pp. 5-12). Lisboa: Texto.

Rendón Rojas, M. Á. (2005) Bases teóricas y fllosóficas de la bibliotecología. México: UNAM, CUIB.

Rendón Rojas, M. Á. (2008). Ciencia bibliotecológica y de la información en el contexto de las ciencias sociales y humanas. Epistemología, metodología e interdisciplina. Investigación bibliotecológica, 22(44), 65-76. Recuperado de http://www.scielo.org.mx/pdf/ib/v22n44/v22n44a4.pdf 
Rendón Rojas, M. Á. (2013). El objeto de estudio de la bibliotecologia/documentación/ciencia de la información: propuestas, discusión, análisis y elementos comunes. México: UNAM, Instituto de Investigaciones Bibliotecológicas y de la Información.

Rendón Rojas, M. Á. (2016). El diálogo entre la archivistica, la bibliotecología, la ciencia de la información y la museología. Un ejercicio intra, inter, multi y transdisciplinario. In: Encontro de Diretores, 11 e Encontro de Docentes de Escolas de Biblioteconomia e Ciência da Informação do Mercosul, 10. Anais eletrônicos. Belo Horizonte: UFMG. Recuperado de http://mercosur2016.eci.ufmg.br/wp-content/uploads/2016/10/AnaisMercosur5.pdf

Silva, A. M. (2009). Arquivologia e gestão da informação / conhecimento. Informacao \& sociedade, 19(2), 47-52.

Silva, A. M. (2013). A ciência da informação e a transição paradigmática. En M. A. Rendón Rojas. (coord.) El objeto de estudio de la bibliotecología/documentación/ciencia de la información: propuestas, discusión, análisis y elementos comunes. México: UNAM, Instituto de investigaciones Bibliotecológicas y de la Información.

Silva, A. M. (2015). Arquivo, biblioteca, museu, sistema de informação: em busca da clarificação possível. Cadernos $B A D, 1,103-124$ Recuperado de https://www.bad.pt/publicacoes/index.php/cadernos/article/viewFile/1482 /pdf_7

Silva, A. M. (2017). Que ciência da informação precisamos para enfrentar a complexidade? PontodeAcesso, Salvador, 11(1), 85-114. Recuperado de https://www.pontodeacesso.ici.ufba.br

Silva, A. M. e Ribeiro, F. (2010). Paradigmas, serviços e mediaçôes em ciência da informação. Recife: Nectar.

Tanus, G. F. (2016). A constituição da biblioteconomia científica: um olhar histórico. RDBCI: Revista digital de biblioteconomia e ciência da informação, 14(2), 217-231 http://dx.doi.org/10.20396/rdbci.v14i2.8643878

Vega-Almeida, R.L., Fernández-Molina, J. C. \& Linares Columbié, R. (2009). Coordenadas paradigmáticas, históricas y epistemológicas de la Ciencia de la Información: una sistematización. Information research, 14(1). Recuperado de http://InformationR.net/ir/14-1/paperxxx.html]

\section{Notas}

1 Se graduó de filosofía en la Faculdade de Filosofía de la Universidade Católica Portuguesa de Braga; y de Historia en la Faculdade de Letras de la Universidade de Porto. Graduado de bibliotecario-archivista por la Faculdade de Letras de la Universidad de Coimbra. Es Doctor en Historia Contemporánea por la Universidade de Minho. Actualmente, es profesor de la licenciatura, maestría y doctorado de la Universidade de Porto. Es autor de diversas obras referidas al estudio de la ciencia de la información y la archivística.

2 Es graduado de filosofía en la Universidad Estatal de Moscu "M. V. Lomonósov" en Rusia en el año 1987, donde también concluyó su Maestría y Doctorado en los años 1989 y 1993, respectivamente., especializándose en el área de lógica. Actualmente, es profesor e investigador del Instituto de Investigaciones Bibliotecológicas y de la Información de la Universidad Nacional Autónoma de México (UNAM). 